\title{
Comunicación positiva contra la desigualdad en América Latina: Anita Garibaldi - TECHO
}

\author{
Positive communication against inequality in Latin America: Anita \\ Garibaldi - TECHO
}

\author{
Leonardo BAEZ COLEGIO \\ Colegio Universitario de Periodismo (Argentina) \\ Alejandro ÁLVAREZ-NOBELL \\ Universidad Nacional de Córdoba (Argentina)
}

Recibido: 3 de febrero de 2016

Aceptado: 07 de marzo de 2016

\section{Resumen}

En el marco de la Copa Mundial de Futbol, Brasil 2014, la ONG "TECHO" llevó adelante la campaña "Anita Garibaldi, el desafío al campeón del mundo". Este estudio analiza la estrategia y los resultados desde los enfoques de la comunicación positiva (Muñiz y Álvarez, 2013), mediante la cual la campaña procuró dejar al descubierto la pobreza que existe en Brasil y Latinoamérica, y que nadie muestra, apelando a modelos de persuasión positiva, con mensajes que fomentan consciente o inconscientemente la felicidad y el bienestar psicológico de los públicos a los que se dirigió. La misma involucró múltiples acciones, obteniéndose con ella un alcance de 10.603 .187 millones de personas; 30.738 visitas en los videos de youtube, 308 impactos en prensa internacional y 22 influenciadores apoyando la campaña, entre ellos CALLE 13. El aporte al bienestar positivo se buscó desde la construcción de un storytelling que tomó como eje un tema que "nos iguala" a todos, como es el fútbol.

\footnotetext{
Abstract

In the framework of the World Cup Soccer, Brazil 2014, the NGO "ROOF" carried out the campaign "Anita Garibaldi, challenge the world champion." This study analyzes the strategy and results from positive communication approaches (Muñiz and Alvarez, 2013), by which the campaign sought to expose the poverty in Brazil and Latin America, and nobody shows 
appealing to models positive persuasion, with messages that consciously or unconsciously promote happiness and psychological well-being of the public to whom it is addressed. It involved multiple actions, giving her a range of 10,603,187 million people; 30,738 views on youtube videos, 308 impacts on international media and influencers 22 will support the campaign, including CALLE 13. The contribution to positive well was sought from building a storytelling that took centered around an issue that "we equals" to all as is football.

Palabras claves: Comunicación Positiva; felicidad; desigualdad; tercer sector; TECHO, pobreza, asentamientos informales.

Keywords: Positive Communications; happiness; inequality; third sector; TECHO, poverty, informal settlements.

\title{
1. Introducción
}

Al margen (o contrariamente, quizás) de la riqueza, el crecimiento material y económico que ha marcado el afán de vida de personas y países durante tantas décadas; en 2012, el concierto de países del mundo reunidos en la Asamblea General de Naciones Unidas declararon que "la felicidad y el bienestar son objetivos y aspiraciones universales en la vida de los seres humanos de todo el mundo"'.

Sin duda, el propósito de esta nueva ecuación fue despejar a los seres humanos como un "recurso" más que media para alcanzar el bienestar económicos y materiales, el cual se ha vueltos desde hace ya demasiado tiempo un fin en sí mismos. Y más allá de su claro afán declarativo y (re)fundante, es de esperar que traiga consecuencias en las políticas de los países y también en el resto de organizaciones de todos los sectores. En ese sentido, son cada vez más numerosas las evidencias científicas que se suman a las que ya existían intuitivamente de que no parece que la abundancia material traiga a priori el bienestar de las personas (Kasser y Sheldon, 2009). Y es que aunque egoísmo y solidaridad (como mecanismos de supervivencia personal y social) no acaban de encontrar el justo equilibrio, cada vez resulta más evidente que el progreso humano (Ridley, 2011) se sustenta en el

\footnotetext{
${ }^{1}$ [Disponible en http://www.un.org/es/events/happinessday/] Consultado el 10/03/2015
}

\author{
Facultad de Ciencias Políticas, Sociales y de la Comunicación \\ Universidad de La Laguna \\ Avenida César Manrique, s/n; Campus de Guajara \\ 38071 La Laguna, Tenerife (Islas Canarias - España)


intercambio basado en el principio de confianza mutua, interdependencia, cooperación y comunicación.

Bajo estos principios, desde 1997, TECHO ${ }^{2}$ lleva trabajando en Latinoamérica (actualmente con proyectos en 19 países de la región e incidencia en 451 asentamientos informales de Latinoamérica) por paliar la desigualdad, y contribuir proactiva y positivamente al desarrollo humano. TECHO es una organización internacional de la sociedad civil cuya misión es superar la situación de pobreza en asentamientos informales que viven miles de personas; frente a una problemática, la de la desigualdad en América Latina, que estructuralmente genera pobreza y exclusión, pese al crecimiento de la última década en la región.

Para abordar esta compleja problemática, TECHO ha diseñado un plan estratégico de comunicación basado en el desarrollo de competencias actitudinales y emocionales de las personas y claramente alineado con el mandato que la Global Alliance for Public Relations and Communication Management ${ }^{3}$ suscribió en 2012 en el marco del Foro Mundial de Relaciones Públicas (Mandato de Melbourne: un llamado a la acción para nuevas áreas de valor en las Relaciones Públicas y la administración de la comunicación), en el cual sostiene, entre otros aspectos de relevancia, la necesidad de "reforzar una cultura de mejora organizacional involucrando a los públicos internos y externos en un diálogo significativo y un cambio positivo" (Global Alliance, 2012:8).

En particular, objeto de esta comunicación, nos hemos propuesto reflexionar sobre la campaña de comunicación "Anita Garibaldi, el desafío al campeón del mundo Brasil 2014"4 que TECHO llevó adelante con el objetivo de visibilizar la problemática que se vive en los asentamientos informales de Latinoamérica.

Anita Garibaldi es una favela (de las más de 6.300 que existen en Brasil) fundada hace 13 años en Guarulhos, Sao Pablo y en la viven actualmente casi 4.000 familias, con historias

\footnotetext{
${ }^{2}$ [Disponible en http://www.techo.org] Consultado el 10/02/2015

3 [Disponible en http://www.globalalliancepr.org] Consultado el 14/02/2015

${ }^{4}$ [Disponible en http://www.techo.org/desafioanita/index_es.html] Consultado el 10/02/2015
}

Facultad de Ciencias Políticas, Sociales y de la Comunicación Universidad de La Laguna Avenida César Manrique, s/n; Campus de Guajara 38071 La Laguna, Tenerife (Islas Canarias - España) 
marcadas por el sacrificio, trabajo conjunto, cientos de adversidades, incluyendo la represión policial. En 2003 fundaron su propio equipo de fútbol para contribuir a la comunidad y que a través del deporte lograran mostrar su realidad. Además, cuentan con una academia de fútbol con 150 niños de la comunidad, allí además de fútbol se practican los valores como el respeto y el trabajo en equipo. Para participar los niños deben obtener buenas clasificaciones en su Escuela, fomentando así su educación formal. Desde hace 13 años que en esta comunidad no se trabaja para ser los mejores, sino para ser cada vez mejores, siempre trabajando juntos.

La campaña procuró lograr el aporte al bienestar positivo desde la construcción de un storytelling que tomó como eje un tema que "nos iguala" a todos, como es el futbol. Además buscó posicionar a las personas en situación de pobreza desde sus capacidades de resiliencia, con un enfoque transformador desde la acción y no como se los suele mostrar en los medios de comunicación masiva.

En efecto, con este análisis procuramos entonces comprender los modos en el que la comunicación positiva se vuelve una estrategia organizacional contra la desigualdad, en post del bienestar y el progreso humano. Para ellos partimos de las siguientes hipótesis:

H1: Los personajes del documental se presentan como individuos que experimentan la felicidad y el bienestar personal, provocando con sus conductas un impacto emocional comunicativo positivo en los públicos.

H2: El empleo de recursos discursivos basados en la acción y los valores son fácilmente transmisibles en las distintos medios y acciones empleados para la campaña, lo cual contribuyen a lograr un impacto emocional comunicativo positivo.

\section{Asentamientos Informales en América Latina. Desigualdad, exclusión y pobreza}

Facultad de Ciencias Políticas, Sociales y de la Comunicación Universidad de La Laguna Avenida César Manrique, s/n; Campus de Guajara 38071 La Laguna, Tenerife (Islas Canarias - España) 
El $80 \%$ de la población latinoamericana vive en ciudades. Después de décadas de acelerado crecimiento demográfico, la región ha llegado a ser considerada la más urbanizada del planeta. Como resultado de un proceso acelerado y desordenado de crecimiento, en estas ciudades uno de cada cuatro habitantes es poblador de un asentamiento informal en situación de pobreza.

Asimismo, es bastante conocido que actualmente la región es la más desigual del mundo. En gran medida, esto ha sido el resultado de décadas de haber implementado políticas neoliberales que apostaban por el crecimiento económico a partir de la apertura de los mercados en base a una lógica privatizadora que llegó a incluir la prestación de servicios públicos. Entre otras cosas, esto terminó desmantelando los incipientes sistemas de seguridad estatal latinoamericanos o impidiendo su construcción de hecho.

Actualmente en las ciudades de Latinoamérica, según datos de CEPALSTAT (2015), el 10\% de la población más rica acapara aproximadamente el 37\% de los ingresos totales, esto es casi el doble del ingreso total de la mitad más pobre de la población urbana de la región, que recibe un $20 \%$ de los ingresos.

El caso de Brasil es todavía más extremo, el $10 \%$ de mayores ingresos representa aproximadamente el $48 \%$ del ingreso disponible en las ciudades y el $50 \%$ más pobre $16 \%$. Es decir, la élite de las ciudades (10\% más rico) recibe un ingreso 3 veces mayor que las mayorías urbanas empobrecidas ( $50 \%$ más pobre).

Los altos niveles de desigualdad en territorios predominantemente urbanos, han dado como resultado ciudades caracterizadas por las dinámicas de profundas segregación. En un extremo se encuentran los más excluidos, que se aglomeran en territorios con bajo valor de mercado y con severas limitaciones para acceder de manera adecuada a servicios públicos. Espacios que muchas veces han tenido que ser ocupados y defendidos de diversas 
intenciones de desalojo. $Y$ que se encuentran principalmente en los espacios abandonados por la dinámica económica en las zonas de centrales de las ciudades o, como es el caso de Anita Garibaldi, en las marginadas periferias urbanas.

Por el otro lado, la segregación también ser refleja en la autoexclusión de las élites de mayores ingresos. Generalmente en lugares periféricos de alto valor comercial, donde se edifican lujosas zonas residenciales con acceso a múltiples servicios y espacios privados para uso exclusivo de sus residentes.

La segregación genera el fracturamiento de las conexiones entre los habitantes de las ciudades. Esto refuerza el debilitamiento de los espacios públicos que sirven como plataformas para la promoción del tejido social urbano. En general, estos espacios han cedido su lugar a centros comerciales privados de recreación y consumo. En el caso de los territorios con mayores niveles de pobreza, los espacios públicos se limitan a algunos espacios precarios disponibles en las comunidades, como vendría siendo la cancha de fútbol con limitadas instalaciones de la comunidad Anita Garibaldi.

Los países latinoamericanos no han sido capaces de generar ciudades armónicas donde se promueva el desarrollo de los habitantes en condiciones de igualdad y respeto a los derechos. Por el contrario, las ciudades se han convertido en las más peligrosas del mundo y se caracterizan por los altos niveles de segregación social y territorial. Se ha priorizado la generación de condiciones para promover la actividad económica privada y la comodidad individual prevalece por sobre el bienestar común.

Las exclusión social producto de la desigualdad está asociadas directamente a la vulneración de los derechos de una parte importante de la población. La pobreza, en el contexto de estas ciudades, es una manifestación extrema de las dinámicas de desigualdad que rigen las 
relaciones socioeconómicas de las sociedades y que tiene directas implicaciones en las dimensiones políticas, culturales, educativas, etc. que condicionan la calidad de vida presente y futura de los pobladores de los asentamientos informales.

En ese sentido, si bien la proporción de la población urbana viviendo en situación de pobreza dentro de la región ha descendido en la última década, en coincidencia con una leve e insuficiente reducción de la desigualdad, en la actualidad hay 124 millones de personas viviendo en esta situación. Aproximadamente un tercio de estas personas viven en Brasil.

Esta es una problemática que se percibe en la vida cotidiana de las ciudades latinoamericanas. Entre distintos tipos de desigualdades, los habitantes de la región consideran que la desigualdades socieconómica es uno de los factores que producen mayores conflictos en las relaciones sociales. Según un estudio de UN-Habitat (2012), principalmente son las desigualdades derivadas del antagonismo entre pobreza - riqueza, las cuales para aproximadamente el $80 \%$ de los encuestados es una causa fuerte de conflicto social.

En línea con los argumentos anteriores, este antagonismo entre pobreza y riqueza económica, dentro de los modelos de mercado altamente privatizados, tiene repercusiones directas en las desigualdades de acceso a educación, salud, vivienda, entre otros. Con respecto a la segregación espacial de las ciudades latinoamericanas, dos tercios de los pobladores consideran que los barrios ricos o pobres son los que generan más desigualdades.

Ante a esta percepción de las magnitudes de la desigualdad y sus consecuencias, la encuesta de UN-Habitat (2012) expone que el $84 \%$ los habitantes de las ciudades de la región considera que avanzar en generar mayores niveles de igualdad socioeconómica 
aporta a la construcción de la democracia. Para el caso de la ciudad de Sao Paulo, donde está ubicado Anita Garibaldi, este dato aumenta al 87\%. Esta puede ser considerada una demanda socialmente aceptada por la igualdad.

\section{Aportar a la construcción de un nuevo sentido común}

Frente a una emergencia social con base en la desigualdad de las dimensiones expuestas anteriormente, el trabajo de $\mathrm{TECHO}$ se enmarca en un esfuerzo regional por aportar al desarrollo de una conciencia social común basada en un conjunto de ideas, valores y argumentos que aporten a entender los déficits estructurales de las sociedades de la región y, a partir de allí, incentivar el desarrollo de acciones que busquen superar dichas deficiencias.

Si bien es cierto, el cumplimiento de esta misión no se limita a las acciones de comunicación de TECHO, sin lugar a dudas la estrategia comunicacional tiene un papel preponderante. Para garantizar que todas las acciones institucionales y en amplia colaboración con otros actores, aporten al tremendo desafío social por construir colectivamente un nuevo sentido común que permita entender el presente latinoamericano y desde allí trabajar por la transformación social de nuestra región.

En ese sentido, se busca concientizar críticamente sobre la realidad así como también se invita a proyectar con optimismo un futuro diferente. En línea con el análisis de Judith Butler (2014), sobre el proceso dinámico de conformación del pueblo como sujeto social. A través de la promoción de contenidos y puesta en práctica de acciones que fomenten las relaciones de igualdad y la solidaridad, por ejemplo el voluntariado, la organización comunitaria, etc,. TECHO busca aportar a introducir en el mundo dinámicas de igualdad social que 
aparentemente están ausentes o son deficitarias en la realidad actual y se consideran fundamentales para un nuevo futuro.

Desde una perspectiva de promoción de la acción ciudadana, concepto que es en esencia igualdad social. A grandes rasgos, en un contexto dominado por la lógica del mercado y frente a la desigualdad y la segregación, TECHO se suma a la promoción de la igualdad de derechos. Ante la hegemonía de intereses privatizados, se propone la colaboración recíproca. En contraposición de la dinámica de precios y el lucro, la acción colectiva solidaria y gratuita. Frente a un modelo de sociedad basado en el individualismo, la invitación a la búsqueda del bien común.

Si bien es cierto, las bases de la transformación social requieren de un proceso que tiene como actor fundamental a los grupos subordinados en la realidad actual. En línea con la perspectiva de participación, TECHO considera que todos los sectores sociales pueden jugar un papel en este proceso.

Existe la confianza que sociedades más igualitarias, donde todos sean sujetos de derechos y nadie sea víctima de la pobreza producto de la exclusión social, son modelos sociales más beneficiosos para el conjunto de sus habitantes. Por lo tanto, su búsqueda y construcción puede ser una responsabilidad compartida $y$, aunque es un proceso que contiene conflictividades, finalmente tiene un impacto directo y positivo en todos los miembros de la sociedad.

En ese sentido, se apuesta por contribuir a la construcción de un movimiento diverso, multitudinario y amplio, que en su recorrido dinámico experimente las alegrías de avanzar hacia el futuro conquistando derechos, construyendo igualdad y cambiando la realidad.

\section{Comunicación Positiva para el bienestar y el desarrollo humano}

La felicidad no es algo que ocurra, es algo que se construye y la comunicación es la mejor herramienta. Mora sostiene que la felicidad no deja de ser un constructo mental de 
sentimiento y pensamiento, y que para existir "precisa de un lenguaje y unos niveles de pensamiento y sentimientos bastante sofisticados, y requiere de un cerebro con niveles de conciencia que solo se alcanzan en escenarios de interacción social alta, de comunicación fluida" (2012: 38). Parece, por tanto, que la felicidad necesita ineludiblemente de la comunicación para existir. Y si necesita de la comunicación, necesita de la persuasión positiva, porque toda comunicación lleva algo de persuasión, ya que comunicar es influir (Watzlawick, 1993).

Así es como la comunicación positiva (Muñiz y Álvarez, 2013), paradigma incipiente importado de los planteamientos sobre Psicología Positiva (Seligman, 1998) se define como aquella comunicación organizacional que, al margen de otros objetivos, fomentar consciente o inconscientemente la felicidad y el bienestar psicológico de los públicos a los que se dirija. las estrategias de relaciones con los públicos ponen el foco no en estereotipos negativo de las disfunciones humanas, como era tradicional, sino en lo positivo, en el estado óptimo de la existencia humana y en los caminos por alcanzarlo.

Llevado al terreno de la gestión de comunicación en las organizaciones o Relaciones Públicas, desde una perspectiva contemporánea, simétrica y bidireccional (Grunig, 2000) los supuesto enunciados se deberían acercar de manera natural al planteamiento que defendemos. Según Magallón, las relaciones públicas procuran el "establecimiento de vínculos plausibles y deseados entre la organización en cuestión y sus públicos específicos" (2006: 104), a lo que Wilcox, Cameron y Xifra sumaran que implican "la construcción de relaciones mutuamente beneficiosas entre la organización y sus diversos públicos (...) en las que todas las partes ganan" (2006: 8).

Con lo cual, hablaremos de Relaciones Públicas positivas o comunicación positiva en las organizaciones cuando sus fines superan la consecución no ya de los intereses mutuos (de organizaciones e individuos), sino de aquellos intereses encaminados al bienestar y la felicidad.

Facultad de Ciencias Políticas, Sociales y de la Comunicación Universidad de La Laguna Avenida César Manrique, s/n; Campus de Guajara 38071 La Laguna, Tenerife (Islas Canarias - España) 
En este marco, seleccionamos un conjunto de variables de estudio: en primera medida se ha procurado identificar el insight social (Romero y Sánchez, 2012) presente y el mecanismo revulsivo inconsciente que contiene, así como la presencia o no de atributos (a modo de valores) que promueven el bienestar y la felicidad. Asimismo, se han caracterizado a los personajes del documental, sus modos de representación en vinculación con la felicidad y el bienestar personal; y como consecuencia el impacto emocional comunicativo positivo que pueden provocar en los públicos.

Finalmente analizamos el empleo de recursos discursivos basados en la acción y los valores que son transmisibles en las distintos medios y acciones empleados para la campaña, y su contribución para lograr también un impacto emocional comunicativo positivo.

\section{Método y caso de estudio}

Analizar la contribución de la comunicación positiva en las estrategias organizacionales de TECHO frente a la desigualdad en América Latina, en particular Brasil, implica enmarcar tales estrategias como una respuesta creativa a un sentimiento generalizado de desesperanza en una sociedad afectada por tal situación. Para ello, circunscrito en un diseño metodológico exploratorio descriptivo, esta investigación social, procuró verificar mediante la interpretación de los resultados de la campaña a la luz de las variables en cuestión, la corroboración de las hipótesis teóricas propuestas.

El análisis cualitativo se basó en el estudio de los personajes protagonistas, sus características más relevantes así como sus comportamientos, sus relaciones y las acciones mostradas en las piezas. En segundo lugar, se han observado los recursos discursivos tanto en la expresión verbal como en las imágenes. Finalmente se han identificado los valores, tanto humanos como organizacionales transmitidos en la campaña. 
La campaña, denominada "Anita Garibaldi, el desafío al campeón del mundo", se desarrolló en el marco de la Copa Mundial de Futbol, Brasil 2014, entre los meses junio y julio de 2014 y contó con el apoyo de las agencias de comunicación Young and Rubicam (creatividad), Patria (realización) y Burson Marsteller (estrategia comunicacional). Además, contó con el apoyo de medios de comunicación del mundo, agencias de noticias, embajadores como el grupo musical Calle 13, entre otros.

Consistió básicamente en la elaboración de un documental por el cual se desafiaba al próximo campeón mundial a jugar un partido contra Anita Garibaldi FC, el equipo de la favela de San Pablo (Brasil) que lleva el mismo nombre, en el marco de un concierto de acciones que apoyaban los objetivos de la misma: gestión de prensa con visitas a la comunidad, difusión de embajadores en social media, participación de Anita Garibaldi en eventos deportivos dentro de la Copa del Mundo.

Más allá de los propios resultados obtenidos por la campaña, el estudio que aquí se presenta es producto de un acercamiento exploratorio a una realidad de difícil e interesante observación. Esta temática de investigación, ya viene siendo objeto de trabajos científicos de mayor envergadura, nutridos de los aportes teóricos y trayectos que como tal ha desarrollado el grupo de investigación Felicicom Lab (Laboratorio de comunicación organizacional, persuasión y felicidad) durante 2011 a 2013 y que actualmente continúa el grupo "COMINEL: Comunicación Estratégica y Emocional' con sede en la Facultad de Comunicación de la Universidad San Jorge (España).

\section{El desarrollo de la campaña y sus acciones}

Vázquez (en Avia y Vázquez, 2011) afirma que el optimismo es hoy y ante todo una exigencia ética, para con nosotros mismos, y para con los demás. En esta línea, TECHO define en sus políticas de comunicación que los mensajes deben estar "vinculados a enfoque esperanzador de la pobreza: posicionando a las personas que viven en situación de pobreza 
como sujetos de derecho, como actores que están transformando su realidad desde todas las capacidades que ellos tienen, y nunca desde sus carencias".

Bajo estos precepto, campañas como la que es objeto de este análisis se suceden continuamente con el objetivo final de dejar al descubierto la pobreza que existe en Brasil y Latinoamérica, y que nadie muestra. Analicemos a continuación los principales aspectos y resultados de la misma.

Durante el desarrollo de la campaña, TECHO buscó generar una estrategia 360 grados: videos en youtube, gestión de prensa, participación en eventos de fútbol en Brasil, acciones en social media, comunicación interna, entre otros. Debido al contexto de la Copa del Mundo, la alta competencia de medios y los escasos recursos que tienen las ONGs para posicionar mensajes en los medios de comunicación, TECHO avanzó con estrategias que no demandaban alta inversión. La mayor complejidad sin duda fue captar la atención de los públicos, durante el acontecimiento mundial que más impacto genera en la sociedad. La campaña generó resultados significativos tanto hacia los grupos de interés internos como para los externos. En cuanto a lo interno, permitió visibilizar y desarrollar un proyecto para afianzar el nuevo discurso de la marca (antes Un Techo para mi País) con una propuesta sumamente creativa. El proyecto logró un alcance de 10,6 millones de personas en internet y social media. La historia de Anita Garibaldi FC llegó a 50 países por medio de 250 medios que realizaron coberturas periodísticas de esta historia.

\subsection{Las estrategias de comunicación positiva}

La campaña tubo por insight social mostrar la pobreza desde sus atributos, sus capacidades, con historias comunes y con temas "comunes" que movilizan a la sociedad en general, empleando para ello una temática como es el fútbol, y su manera de contribuir al bienestar y a la felicidad.

Facultad de Ciencias Políticas, Sociales y de la Comunicación Universidad de La Laguna Avenida César Manrique, s/n; Campus de Guajara 38071 La Laguna, Tenerife (Islas Canarias - España) 
Entre las particularidades de este proyecto se encuentra el enfoque con el que fue concebido la figura de las "personas beneficiadas": se mostró la alegría y entusiasmo con la que viven sus desafíos pese a vivir en situación de exclusión y con sus derechos vulnerados. Sin duda estos modos de representación fueron claves para lograr el gran impacto emocional comunicativo positivo en los públicos que fueron destinatarios finales de la campaña.

"Yo soy un representante de mi equipo y mi comunidad. Esto nos sirve para levantar nuestro nombre con orgullo y mostrar lo que hemos hecho, lo que estamos realizando y lo que queremos para lograr que nuestras vidas sean mejores", declaró Orley Fereiras, uno de los jugadores del equipo de Anita Garibaldi. Así, los 16 jugadores del equipo que representó el asentamiento lanzaron su desafío por las redes sociales de la organización para visibilizar la situación en la que viven y su trabajo para superarla. La historia de esta favela alcanzó los 19 mil fans solo en su sitio web, equivalente a la mitad de los tickets vendidos a la hinchada chilena en este Mundial 2014.

Pese al riesgo constante en que viven los 15 mil habitantes de esta favela -privados del acceso regular a luz, agua y saneamiento- la comunidad se organizó para alcanzar a las selecciones ganadoras. A través de una invitación oficial, TECHO se acercó a las federaciones de Alemania y Argentina además de las de Brasil y Holanda como semifinalistas. Así, el mensaje definido por TECHO, y que los personajes representaron en el documental decía: "No vamos a descansar hasta lograr el desafío de que el campeón del mundo juegue con Anita. No es un imposible, porque sabemos que el equipo y la comunidad están acostumbrados a enfrentar dificultades para mejorar su situación. Anita Garibaldi es un ejemplo de esfuerzo y trabajo conjunto, que representa a las 113 millones de personas que viven en asentamientos informales en Latinoamérica".

En cuanto a los recursos discursivos, medios y acciones empleados para la campaña, para este proyecto, se generó un documental de 3 minutos, formato que permitió contar la historia desde la comunidad y desde adentro, pero dando un claro contexto en el cuál se desarrolló 
la iniciativa. Los medios utilizados para la campaña fueron: social media institucionales de TECHO, correo electrónico, relaciones con la prensa, medios internacionales, embajadores que apoyaron la campaña en sus redes sociales personas, participación en eventos deportivos. La historia de Anita dio la vuelta al mundo y fue publicada en espacios de los 5 continentes. Fue transmitida en más de 50 países y en 250 medios distintos, entre los que destacan Fox, Buzzfeed, ESPN, El País, Televisa y las agencias France Press y EFE.

\section{Reflexiones finales}

La campaña, en términos de comunicación, apelando a la persuasión positiva y mediante el empleo de un mensaje que fomente consciente o inconscientemente la felicidad y el bienestar psicológico de los públicos a los que se dirigió cumplió sus objetivos ampliamente. En sus múltiples acciones, términos de impacto, la misma permitió llegar a 10.603.187 millones de personas, 30.738 visitas en los videos de youtube, 308 impactos en prensa internacional y 22 influenciadores apoyando la campaña, entre ellos CALLE 13.

En cuanto al objetivo propuesto en esta investigación, mediante la cual procuramos comprender los modos en el que la comunicación positiva se vuelve una estrategia organizacional contra la desigualdad y en post del bienestar y el progreso humano, podemos afirmar, respecto de las hipótesis planteadas:

Que la primera hipótesis, que afirmaba que los personajes del documental, al presentarse como individuos que experimentan la felicidad y el bienestar personal, han provocado efectivamente conductas con un impacto emocional comunicativo positivo en los públicos. Está más que claro que la estigmatización o representación de aspectos negativos de la situación de vulnerabilidad no formaron parte de la estrategia.

Con respecto a la afirmación propuesta con respecto a que el empleo de recursos discursivos basados en la acción y los valores son fácilmente transmisibles en las distintos medios y acciones empleados para la campaña, lo cual contribuyen a lograr un impacto 
emocional comunicativo positivo, los impensables niveles de exposición, en un ecosistema discursivo altamente saturado (por el Mundial) dan cuenta del éxito de la campaña en este sentido.

Sin duda, el aporte al bienestar positivo se buscó desde la construcción de un storytelling que tomó como eje un tema que "nos iguala" a todos, como es el fútbol. Con ello, TECHO ha procurado también lograr un impacto positivo en cuanto a la consecución no ya de los intereses mutuos de visibilidad, básicamente (de la organización y de la favela), sino de aquellos intereses encaminados al bienestar y la felicidad de las personas y el colectivo vulnerable. En definitiva, estrategias como la analizada permiten posicionar a las personas en situación de pobreza desde sus capacidades de resiliencia, con un enfoque transformador desde la acción y no como se los suele mostrar en los medios de comunicación masiva.

\section{Enlaces}

- Documental: Anita Garibaldi "El desafío al Campeón del mundo" (TECHO): [https://www.youtube.com/watch?v=Pt03gFeV4Pc\&list=PLzmrpVAazjA aAQmIOoBbp3DwTcF8Sug]

- Microsite de la Campaña (TECHO): [http://www.techo.org/desafioanita/index es.html]

- Reportaje "La favela que desafía al campeón de Brasil 2014" (El País - Cali) [https://www.youtube.com/watch?v=adzEdJdgknU]

\section{Referencias}

Álvarez Nobell, A. y Muñiz Velázquez, J. A. (2013). Felicidad y desarrollo de la Cultura en las organizaciones, un enfoque psicosocial en Revista de Comunicación, 12, 7.

Avia, M.D. y Vázquez, C. (2011). Optimismo Inteligente (2ª ed.). Madrid: Alianza.

Global Alliance (2012): El mandato de Melbourne. Un llamado a la acción para nuevas áreas de valor en las Relaciones Públicas y la administración de la comunicación. FORO 
MUNDIAL DE RELACIONES PÚBLICAS. Melbourne: Global Alliance. Disponible: http://melbournemandate.globalalliancepr.org/

Grunig, J. y Hunt, T. (2000). Dirección de relaciones públicas. Edición adaptada por Jordi Xifra. Madrid: Gestión 2000.

Kasser, T. y Sheldon, K. M. (2009). Time affluence as a path toward personal happiness and ethical business practice: empirical evidence from four studies. Journal of Business Ethics, 84, p. 243-255.

Lyubomirsky, S., Rey, L. y Diener, E. (2005). Los beneficios de la frecuente afecto positivo: ¿Lleva la felicidad del éxito? Psychological Bulletin, 131 (6), 803-855.

Magallón, S. (2006). Concepto y elementos de las relaciones públicas. Anàlisi, 34, p. 103109.

Mora, F. (2012). ¿Está nuestro cerebro diseñado para la felicidad? Madrid: Alianza.

Muñiz-Velázquez, J. A., \& Álvarez-Nobell, A. (2013). Comunicación positiva: la comunicación organizacional al servicio de la felicidad. Vivat Academia, (124), 90-109.

Ridley, M. (2011). El optimista racional. Madrid: Taurus.

Romero, M. y Sánchez, M. (2012). Análisis del uso del impacto emocional y la felicidad como recursos publicitarios en tiempos de crisis, en el II Congreso Publiradio, Universidad Autónoma de Barcelona.

Seligman, M. (2004). Aprenda optimismo. Barcelona: Debolsillo.

Seligman, M. E. P. (2004). Aprenda optimismo. Barcelona: Debolsillo.

Seligman, M.E.P. (2011). La vida que florece. Barcelona: Ediciones B.

Watzlawick, P. (1993). Teoría de la comunicación humana. Barcelona: Herder.

Wilcox, d. L., Cameron, G. T. y Xifra, J. (2006). Relaciones Públicas. Estrategias y tácticas. Madrid: Pearson.

\section{Forma de citar este artículo en bibliografías}

BAEZ, L. y ÁLVAREZ-NOBELL A. (2016): "Comunicación positiva contra la desigualdad en América Latina: Anita Garibaldi - TECHO", en Revista PANGEA № 7, Volumen № 1, páginas 1 a 17. Tenerife: Red Académica Iberoamericana de Comunicación. Recuperado el _ de de 2 de: http://www.revistapangea.org 